\title{
Robotics into the millennium
}

\author{
R Swaminathan, $M$ Wheeler
}

Automation in clinical laboratories is not new. Since the pioneering work of Skeggs in developing continuous flow systems, automated analysers have become standard in almost all laboratories. It will be difficult to imagine a modern clinical laboratory without automation. This form of automation is sometimes referred to as "microautomation." The next stage in automation is the automation of specimen processing and transport of specimens between analyser or workstations. ${ }^{1}$ This is called "macroautomation" or robotics, and typically consists of an inlet unit, sorter, transport system/tracks, automated centrifuge, level detector, bar code reader, decapper, aliquotter, recapper, and so on. This type of system was pioneered by the work of Professor M Sasaki in Japan.

There are many possible reasons to implement a robotic system in a laboratory.

Economic reasons - The cost of health care is rising universally owing to increased expectations, new technology, and the advancing age of the population. In many industrialised countries, hospitals have been closed or merged with neighbouring hospitals in order to reduce cost and to improve efficiency. Many hospital laboratories are also closed and replaced by centralised laboratory services. Macroautomation/robotics is a possible way to improve efficiency and to cope with the high workload in these centralised laboratories. ${ }^{2}$

To reduce errors-Errors in laboratories are a continuous source of problems and they are mainly due to human factors. It is estimated that the error rate in clinical laboratories may be $1-2 \%$, although many errors go unnoticed. Some of these errors have clinical consequences. There are no data on the cost of these laboratory errors. One estimate puts this at $10 \%$ of all errors in hospital care, equivalent to more that $\$ 1.5$ billion annually in the USA. ${ }^{3}$ Most of these errors are at the preanalytical stages of the sample processing. An important incentive for implementation of total laboratory automation is the opportunity it provides to reduce human errors which occur during labelling of specimen, aliquoting, order entry, and so on.

Errors during the analytical stages are mainly "transcription errors." The latter could be reduced or eliminated by direct interface of the analytical instruments with the laboratory information system (LIS).

Improved quality-One factor determining the quality of a laboratory service is the timeliness of results. If the results can be produced at the right time and place, it will have a significant implication on health care cost. With increasing pressure on bed occupancy and attempts to reduce inpatient care, earlier availability of results is now expected. Macroautomation/robotics has the potential to significantly reduce the turnaround time of tests. It has been suggested that the introduction of robotics will improve the overall turnaround time such that an emergency laboratory becomes redundant.

\section{Modifying factors influencing the implementation of robotics}

NEAR PATIENT TESTING/POINT OF CARE TESTING Technology has advanced to such an extent that it is now possible to do many tests at the bedside. These near patient testing/point of care testing devices are disposable devices, ${ }^{4}$ hand held devices, ${ }^{5}$ or bench top analysers, ${ }^{6}$ and they use samples such as whole blood, urine, or saliva. The operating system of the devices is such that they can be operated successfully by non-laboratory staff. ${ }^{7}$ The tests that can be done at the bedside range from electrolytes, troponin, and enzymes, to Helicobacter serology. At present the range of tests available in a particular device or instrument is small, they are relatively slow to perform, and the reagents are expensive. However, some studies have shown that the total cost of the test is lower than laboratory tests, ${ }^{8}$ while the turnaround time is nearly four times faster. ${ }^{9}$ In the future a wide range of tests will become available on many instruments. As the number of different systems and the range of tests increases, prices would be expected to fall. Introduction of near patient testing/point of care testing will have an impact on laboratory workloads. It is estimated that worldwide point of care testing will grow to $\$ 3.5$ billion in the year $2001 .^{10}$ One model of a future laboratory predicts that laboratory workload will decrease by as much as $80 \%{ }^{10}$ and the rationale for robotics will be reduced except in the very large hospital laboratories.

IN VIVO MONITORING AND NON-INVASIVE INVESTIGATIONS

In vivo monitoring of glucose, $\mathrm{pH}, \mathrm{PO}_{2}$, and so on is now possible. The range of tests that can be monitored using in vivo devices will increas $\mathrm{e}^{11}$ and in the acutely ill patient this will be an important method of monitoring necessary analytes such as sodium, potassium, creatinine, $\mathrm{pH}$, bicarbonate, and glucose.

Using techniques such as infrared spectroscopy, attempts are being made to measure analytes in fluid just beneath the skin without the need to collect blood in a syringe. ${ }^{12}$ This type of technique may, for example, be used in the near future for monitoring glucose and glycated haemoglobin. ${ }^{12}$ 
DEVELOPMENTS IN BIOTECHNOLOGY/MOLECULAR BIOLOGY

The field of biotechnology/chip technology and molecular biology is advancing rapidly. Developments in this field will have a profound influence in the laboratory. One example of such technology is the development of microarray chip methodology. ${ }^{13}$ In this system a small volume of sample is enough to analyse hundreds of tests (the tests are based on antibodies or oligonucleotides), and several hundred samples can be analysed in this way in a very short time with one compact instrument. ${ }^{14}$ With the unravelling of the human genome it will be possible to examine DNA for defects that may have adverse effects, using DNA chip technology. ${ }^{15}{ }^{16}$ This technology can also be used to look for "foreign" (viral or bacterial) DNA. In the future many of the bacteriological and viral agents will be detected and quantitated by molecular biology technology using chip technology. ${ }^{17}$ The development of aptamers that are oligonucleotides with the capacity to recognise virtually any class of molecule, with high affinity and specificity, will profoundly influence the diagnostic laboratory. ${ }^{18}$

\section{OTHER TECHNOLOGICAL ADVANCES}

Until recently instrument manufacturers have developed instruments for each of the laboratory disciplines. There is now a growing trend for the development of a single instrument capable of analysing samples for many different tests, crossing the traditional departmental boundaries. With such instruments it will be possible to analyse samples for most of the clinical chemistry tests (including hormones), serology tests (HIV, hepatitis, and so on), other immunology tests, haematinics, and many others. These consolidated instruments also have the flexibility to do "reflex" testing (where a second test is automatically ordered, based on the result of the first test), automatic dilution, and testing of different body fluids. With this type of instrumentation it will be possible not only to consolidate sections of a department but also to bring together sections from different departments.

\section{Options}

It is anticipated that some move to greater automation will take place in every hospital laboratory. The degree of automation, putting aside cost, will depend on the impact of the

Table 1 Examples of the different automation available

\begin{tabular}{|c|c|c|}
\hline Extent of automation & Type of automation & Company \\
\hline Total laboratory automation & Diagnostics-independent system & $\begin{array}{l}\text { Beckman Coulter IDS } \\
\text { Labotix } \\
\text { Lab-Interlink }\end{array}$ \\
\hline Total laboratory automation & Diagnostics associated & $\begin{array}{l}\text { Roche } \\
\text { Bayer }\end{array}$ \\
\hline \multirow[t]{4}{*}{ Partial automation } & Diagnostics associated & $\begin{array}{l}\text { Bayer-LabCell } \\
\text { Konelab Corporation }\end{array}$ \\
\hline & Independent systems & $\begin{array}{l}\text { Tecan } \\
\text { Genesis } \\
\text { Olympus }\end{array}$ \\
\hline & Analytical modular systems & $\begin{array}{l}\text { Bayer Advia IMS } \\
\text { Roche Modular } \\
\text { Abbott Architect }\end{array}$ \\
\hline & Postanalytical & RTS Thurnall \\
\hline
\end{tabular}

corresponding factors (amalgamation of hospital services, closure of hospitals, intake of near patient testing, and so on) on the individual sites.

No matter what options a laboratory chooses, it involves considerable expense and probably a great deal of upheaval. As the payback period of some systems could be as long as 10 years, the advantages and disadvantages of the different instruments have to be considered very carefully. Table 1 lists the alternatives for automation of a laboratory, with examples of some of the manufacturers.

TOTAL LABORATORY AUTOMATION (TLA)

Several companies offer tracking systems linked to dedicated units which will centrifuge, aliquot, sort, and deliver samples to different analysers. Following analysis, samples can be automatically stored in a refrigerated unit, from which specimens can be automatically recalled for further analyses. These companies do not manufacture analysers, so the cost of the system cannot be spread over diagnostic tests. In addition to the cost of the system there is usually a significant cost for the modification of the laboratory. Few laboratories will have the large open space necessary to immediately accommodate TLA with all the necessary amenities. Therefore walls need to be removed and extra wiring required for electrical and computer supplies. The main advantage of these systems is that they do not tie the laboratory down to one diagnostics manufacturer. As long as an analyser is able to sample from specimen tubes on the tracking system, any manufacturer's analyser may be used. This may be an important consideration when choosing an analyser now, with TLA planned for the future. It is anticipated that TLA systems will have a life of at least 10 years, compared with the five years of an analyser. After five years, the laboratory will be able to change the supplier of its main analysers, as long as these are compatible with the tracking system. This is very likely in the future, as manufacturers are directly producing analysers compatible with current tracking systems. It is important therefore, given the long expected use of a TLA system, that the limitations as well as the advantages of the different TLA systems are carefully considered and evaluated before purchase.

The Beckman Coulter IDS system transports samples in pucks which limit the diameter of tube which can be used. In addition a special centrifuge has been designed so that the total centrifugation step is only three minutes. In order to achieve adequate centrifugation, spin-up and braking are of short duration. The latter may cause resuspension of the cells, so a blood tube containing gel is recommended. The suitability of these tubes for some analytes has been questioned, ${ }^{19}$ and some laboratories have decided not to use them. Because of the higher throughput, only one centrifuge will be necessary for many laboratories, whereas other systems use two to four centrifuges for the same workload. Greater flexibility of tube size is offered by the Labotix and Lab Inter-link sys- 
tems. The latter has a holder with holes of several sizes which will accommodate a range of tube sizes and also a slide. Only one specimen can be loaded in each holder, so that the large number of holders required could cause a storage problem. The Labotix system uses a puck system but the sprung prongs on each puck allow a range of tubes to be accommodated. This tracking system is slimmer and lighter than other systems and can pass through a relatively small hole in a wall, which offers the possibility of less building work.

The biggest problem lies in paying for such systems. In some countries where analyses are charged to the customer and where higher throughput, lower turnaround times, and greater efficiency attracts more work, the increased income may pay for the system over a relatively short period ( 2.5 to 5.0 years). ${ }^{2}$ Some laboratories have claimed a payback period of less than two years, although accurate figures are hard to find. In countries such as the United Kingdom, where no charges are made to doctors, funds must come from trust funds, partnerships with industry, grants, or reduction in staff numbers. An increased workload in these countries does not contribute to the purchase of the instrument, and payback periods will be considerably longer.

Various diagnostic companies are now developing TLA systems specifically designed for their own diagnostic analysers. The Bayer LabCell system offers such a tracking system, and Roche has a similar arrangement. A front end system for sample preparation is also available. This arrangement should allow customers to pay for the robotics by a sum added to the cost of the diagnostic reagents. This could be spread over reagents of general clinical chemistry, oncology, endocrinology, and haematinics, so that the additional cost per test would appear small. Automation will lead to a smaller work force, so that money is also moved from staff costs to diagnostic costs. What is urgently needed is some standardisation within the field of robotics, as few if any of the systems are compatible. Diagnostic companies are designing systems that may make payment of robotics easier but reduce the flexibility in the choice of analyser and may mean that future developments, for example instruments employing microarray technology for DNA analysis, cannot be linked to the robotic system.

As more and more local hospitals combine their resources, one centre has to cope with a significant increase in workload. TLA should allow fewer staff to handle much larger workloads owing to the greater efficiency in handling samples (reducing bottlenecks by providing a more even delivery of samples to the analyser).

\section{PARTIAL AUTOMATION}

Problems of high cost and building issues may make TLA impractical initially, and laboratories may choose to introduce partial automation as a first step to total automation. Automation may be conveniently divided into preanalytical, analytical, and postanalytical. Manufacturers of TLA are able to sell partial systems, so that TLA can be introduced in a stepwise manner. Although this may allow some automation in the face of limited funds, it may not be economic with regard to phased alterations to the laboratory. Other manufacturers sell instruments specifically designed to address preanalytical steps only. Examples are the Olympus OLA 1500, which decaps and sorts specimen tubes, the Olympus OLA 4000 which sorts and aliquots, and the Tecan Genesis FE500 and FE1500 systems which centrifuge, decap, aliquot, and sort. The smaller instruments have a footprint of about $2.5 \mathrm{~m}$ and so may be accommodated in most laboratories without extensive changes to the fabric. However, larger instruments such as the Genesis FE1500 are up to $5 \mathrm{~m}$ in length.

Preanalytical systems are said to overcome many of the sampling errors which occur in the laboratory. These include sample mix up owing to aliquoting, and dropped or spilt samples resulting from accidents occurring during manual activities. However, a study in our laboratory (table 2) showed that these errors were very small, and that most errors occurred during data entry. The cost of preanalytical systems is very high (about $£ 300000$ ), so the period of payback may be also very long. Felder ${ }^{20}$ calculated that 2.5 whole time equivalents could be saved with the Beckman Coulter IDS preanalytical system. Table 3 shows estimates of the period of payback of this system related to the number and grade of staff saved. Usually it will be staff of lower grade who will be undertaking the tasks that the preanalytical system would carry out.

\section{CONSOLIDATION}

Automation of the analytical processes has been in place for many years, and chemistry analysers carry out many hundreds of tests a day. A sample transport system linking several instruments together reduces the need for aliqnots, gives more efficient use of each specimen, and provides a more even flow around laboratories and departments. Konelab Corporation have developed a transport system which links several of their analysers together, allowing clinical chemistry, immunochemistry, serology, and therapeutic drug monitoring. The

Table 2 Errors in the laboratory which cause non-return results to the clinician

\begin{tabular}{lll}
\hline Data entry & Wrong destination & $3.5 \%$ \\
& Missed analyte & $0.8 \%$ \\
Aliquotting errors & & $<0.2 \%$ \\
Insufficient sample & & $<0.2 \%$ \\
Dropped or spilt specimen & & $<0.2 \%$ \\
Assay failure & $<0.2 \%$ \\
\hline
\end{tabular}

Table 3 Economics of a pre-analytical system

\begin{tabular}{|c|c|}
\hline \multirow{2}{*}{$\begin{array}{l}\text { Cost of preanalytical system } \\
\text { Savings from losing } 2.5 \text { WTE experienced } \\
\text { technologists }\end{array}$} & $£ 300000$ \\
\hline & \\
\hline Period of payback & \\
\hline $\begin{array}{l}\text { Savings from losing } 4 \text { WTE experienced } \\
\text { technologists }\end{array}$ & \\
\hline Period of payback & 3.0 years \\
\hline avings from losing 4 WTE laborator & \\
\hline Period of payback & 6.0 years \\
\hline
\end{tabular}


transport system is supplied by Clids Automation of Finland. The Bayer Advia Labcell offers similar consolidation of tests with the option of adding haematology testing to the system. For some institutes, where there are separate departments of haematology, clinical chemistry, virology, and immunology, personal concerns over consolidation may have to be overcome. Technologists would have no trouble with consolidation as the same instrument may be employed in difference departments for measurements relating to haematology, endocrinology, and so on.

The work cell arrangement does require a lot of space and therefore significant modification to laboratories, not only to accommodate the transport system but also the number of analysers. Modular systems being developed by Abbott, ${ }^{21}$ Bayer, and Roche offer consolidation of tests on a smaller footprint. These instruments allow chemistry and immunochemistry modules to be "bolted" together. Usually up to four modules can be linked as one operating platform which in itself may produce an instrument about $7 \mathrm{~m}$ in length. Such systems do not allow haematology tests to be accommodated. Workcell and modular systems allow laboratories to negotiate with the diagnostic companies for the cost of the transport system to be spread across the reagent costs. Customers may be concerned about being tied into one company for both their robotics and diagnostics. Customers will find that they may have to compromise on several issues if they are to introduce robotics into their laboratory.

The automation of postanalytical robotic storage of samples is available from several companies but is not likely to be a major consideration of laboratories in the first instance. The major issue is reducing errors of sample handling, smoothing out work flow, and handling an ever increasing workload in the face of continued reductions in budget and staff.

As table 2 shows, automation of sample handling does not address the main sources of error which result in a patient's results failing to reach the clinician. Manual input of data must also be reviewed in order to improve a laboratory's service. In the future many more operations will be carried out on the ward. These will include requesting of analyses with positive patient identification and bedside analysis.

Various systems are being developed where patients receive a watch or wrist band contain-

Table 4 Reasons why a result may not reach the clinician

\begin{tabular}{|c|c|c|}
\hline Stage & Procedure & Error \\
\hline \multirow[t]{6}{*}{ Preanalytical } & Patient request written & $\begin{array}{l}\text { Wrong identification; wrong spelling of name; } \\
\text { tests not entered }\end{array}$ \\
\hline & Blood drawn & $\begin{array}{l}\text { Wrong blood tubes; tube unlabelled or } \\
\text { wrongly labelled }\end{array}$ \\
\hline & Blood sent to laboratory & Sample lost or broken \\
\hline & Data entry & $\begin{array}{l}\text { Sample and request card part company; ID or } \\
\text { name misspelt; tests missed; wrong test } \\
\text { requested; wrong destination entered }\end{array}$ \\
\hline & Centrifugation & Tube broken \\
\hline & Aliquoting & Tube wrongly labelled; tubes mixed \\
\hline \multirow[t]{3}{*}{ Postanalytical } & Analysis & Assay failure; sampling error \\
\hline & Result entry to clinician & $\begin{array}{l}\text { Input error for less automated tests; wrong } \\
\text { destination; result lost in transit }\end{array}$ \\
\hline & Result received & Mislaid; computer failure \\
\hline
\end{tabular}

ing their hospital number. When the phlebotomist takes a blood sample, a small instrument with a bar code reader records the patient's details. The analytes to be measured can either be keyed into the instrument or read in from a bar coded chart. In its most sophisticated form the instrument presents the appropriate bar coded tube. This overcomes the problem of blood being taken into the wrong type of tube and the need for the patient to be rebled. The sample is then sent to the laboratory where the bar code can be read, so that the patient's details and tests can be loaded into the pathology computer. This could be done by loading the sample directly onto a robotic system where the bar code is immediately read and the information transferred to the laboratory computer before the sample is processed. Such a system is being developed by Science Park, RAF, John Radcliffe Biomedical Park, Milan, Italy, in collaboration with several manufacturers.

\section{Conclusions}

Table 4 lists reasons why a result may not reach the clinician. The error may have occurred at the requesting site (general practitioner surgery; phlebotomist on ward or at outpatient clinic), at data entry, during analysis, or after analysis. As shown in table 3, few errors occur once the specimen reaches the laboratory and most occur at data entry. A significant number of results "go missing" when sent out by mail, either internally back to wards or clinics or externally back to other laboratories. It would seem, therefore, that it would be better to invest in bedside requesting and electronic transfer of data to and from the laboratory. Why then do the main changes in laboratories appear to be towards robotics and automation? The development or modification of a single department is easier to manage and to cost than developments affecting several departments and grades of staff and different self interests. Cost savings, particularly in staff numbers, are easier to identify. Also automation is being industry led and offers managers many options.

Thus, as we move into a new millennium there will continue to be greater automation of laboratories, probably with a concomitant overall decrease in the number of staff. There will be a very large uptake of modular systems, particularly in medium sized laboratories, as these offer consolidation of tests with a smaller relative space requirement, less modification to laboratory fabric, and simplification of work practice. They also offer significant automation of tests, without a commitment, either in time or cost, to TLA. An increase in near patient testing, moving work away from the core laboratory, will also reduce the need for TLA.

Current large hospital laboratories, and those developed in the future owing to consolidation of pathology services, will move quickly towards TLA, as the development of near patient testing will have less effect on drawing services away from the core laboratory. A later re-examination of pathology services will draw attention to the fact that it continues to be a significant problem in the successful completion of the cycle of patient $\Rightarrow$ blood sample $\Rightarrow$ 
data entry $\Rightarrow$ analysis $\Rightarrow$ result $\Rightarrow$ patient. Attention will be drawn to the pre- and postanalytical errors and planned investment into bedside requesting, and electronic transfer of results will take place.

The effect of these developments on industry will be towards total service systems, with initially partnerships, and finally mergers, between companies offering solutions to all these stages. This opens the way for partnerships between hospitals and diagnostic companies for the delivery of pathology services, and hence for the semi- or full privatisation of pathology services.

1 Bissell MG, Mountain P. The next stage of laboratory automation. In: Bissell MG, Petersen JR, eds. Automated mation. In: Bissell MG, Petersen JR, eds. Automated integration of clinical labor

2 Bauer S, Bissell MG, Petersen JR, et al. Economics; cost accounting and justification. In: Bissell MG, Petersen JR, eds. Automated integration of clinical laboratories: a reference. Washington DC: AACC, 1998:134-44.

3 Bissell MG. The problem of human errors in the clinical laboratory. In: Bissell MG, Petersen JR, eds. Automated integration of clinical laboratories: a reference. Washington DC: AACC, 1998:134-44

4 Price CP, Thorpe G. Disposable analytical devices for point of care testing. In: Price CP, Hicks JM, eds. Point of care testing. Washington DC: AACC, 1999:17-40.

5 Wilding P, Ciaverelli C. Hand-held sensor system. In: Price CP, Hicks JM, eds. Point of care testing. Washington DC: AACC, 1999:41-66.

6 Kallner A. Bench top technology. In: Price CP, Hicks JM, eds Point of care testing. Washington DC: AACC, 1999:67-98.
7 Maclin E, Mahoney WC. Point of care testing technology. $\mathcal{F}$ Clin Ligand Assay 1995;18:21-33.

8 Keffer JH. Economic considerations of point of care testing. Pathology patterns. Am f Clin Pathol 1995;104:S107-10.

9 Bailey TM. Understanding the economics of bedside diagnostic testing. In: Price CP, Hicks JM, eds. Point of care testing. Washington DC: AACC, 1999:213-32.

10 Felder RA. The distributed laboratory: point of care services with core laboratory management. In: Price CP, Hicks JM, eds. Point of care testing. Washington DC: AACC, 1999:99118 .

11 Smith KM. Non invasive testing technology. Clin Chem 1999;45:1586.

12 MacKenzie HA, Ashton HS, Spiers S, et al. Advances in photoacoustic non-invasive glucose testing. Clin Chem 1999;45:1587-95.

13 Kricka LJ. Miniaturization of analytical systems. Clin Chem 1998;44:2008-14

14 Ekins RP. Ligand assays: from electrophoresis to miniaturized microassays. Clin Chem 1998:44:2015-30.

15 Kurian KM, Watson CJ, Wyllie AN. DNA chip technology. f Pathol 1999;87:267-71

16 Kricker LJ. Nucleic acid detection technologies-labels, strategies and formats. Clin Chem 1999;45:453-8.

17 Hodinka RL. The clinical utility of viral quantitation using molecular methods. Clin Diagn Virol 1998;1:25-47.

18 Jayasena SD. Aptamers: an emerging class of molecules that rival antibodies in diagnostics. Clin Chem 1999;45:162850

19 Suolima E. Stability of fifteen commonly measured hormones and proteins in serum, when stored in plain tubes or gel separator tubules. Proceedings of the UK NEQAS Endocrinology Meeting, Edinburgh, April 1998; 3:224.

20 Felder RA. Modular laboratory robotics and automation. $f$ Int Fed Clin Chem 1997;1:56-60.

21 Wheeler M, Rossi R. Abbott Architect i2000 analyser. An evaluation of the reproductive peptide hormone assays. $\mathcal{F}$ Assoc Lab Automation 1999;4:20-3.

\section{Foundations}

\section{On mortuary assistants....}

......and the corpse-bearers have to go down into those terribly fetid caverns full of decomposing corpses and lay in them the bodies that have been brought there; hence they are exposed to very dangerous diseases, especially to malignant fevers and to sudden death, cachexy, dropsy, suffocative catarrh, and other serious diseases; their faces are always corpse-like and they look ghastly, as men well may who themselves have an appointment with death before long. Could one imagine any surer or more certain way of inviting pestilential diseases than to go down thus into the vaults where they must for some time breathe that foul air?

Chapter XVIII: Diseases of corpse-bearers. De Morbis Artificum (Diseases of Workers), by Bernardini Ramazzini (from the Latin text of 1713) 